\title{
Moxibustion Eases Chronic Inflammatory Visceral Pain In Rats Via MAPK Signaling Pathway In The Spinal Cord
}

This article was published in the following Dove Press journal: Journal of Pain Research

\author{
Yan Huang ${ }^{1,2, *}$ \\ Dan Zhang (D) ${ }^{1,3, *}$ \\ Zhi-Yuan $\mathrm{Li}^{4}, *$ \\ Yan-Ting Yang $\mathbb{D}^{3}$ \\ Li-jie $\mathrm{Wu}^{\prime}$ \\ Ji Zhang' \\ Fang-Yuan $\mathrm{Zhi}^{1}$ \\ Xi-Ying $\mathrm{Li}^{1}$ \\ Zheng $\mathrm{Shi}^{3}$ \\ Jue Hong (iD ${ }^{3}$ \\ Xiao-Peng $\mathrm{Ma} \mathbb{D}^{1,3}$
}

'Yueyang Clinical Medical School, Shanghai University of Traditional

Chinese Medicine, Shanghai 201203,

People's Republic of China; ${ }^{2}$ Acupuncture and Moxibustion Department, Huangpu

District Hospital of Integrated Traditional Chinese and Western Medicine, Shanghai 2000 I0, People's Republic of China; ${ }^{3}$ Laboratory of Acupuncture, Moxibustion, and Immunology, Shanghai Research Institute of Acupuncture and Meridian, Shanghai 200030, People's Republic of China; ${ }^{4}$ Acupuncture and Moxibustion Department, Zhejiang Provincial Hospital of TCM, Hangzhou, Zhejiang 310006, People's Republic of China

*These authors contributed equally to this work

Correspondence: Xiao-Peng Ma Shanghai Research Institute of Acupuncture and Meridian, No. 650 South Wanping Road, Xuhui District, Shanghai, People's Republic of China

Tel +8602I 64690257

Fax +8602I 64382I II

Email pengpengma@163.com
Purpose: The purpose of this study was to explore the central analgesia mechanism of moxibustion for chronic inflammatory visceral pain (CIVP).

Methods: A CIVP rat model was established by 2,4,6-trinitrobenzene sulfonic acid (TNBS) plus $50 \%$ ethanol via enema. The analgesic effect of moxibustion was evaluated using the abdominal withdrawal reflex (AWR), mechanical withdrawal threshold (MWT), and thermal withdrawal latency (TWL). The expression profile of phosphorylated proteins of the mitogen-activated protein kinase (MAPK) signaling pathway in the spinal cord was assayed by protein microarray. The differentially expressed proteins were examined by Gene Ontology (GO) and Kyoto Encyclopedia of Genes and Genomes (KEGG) for functional clusters and corresponding signaling pathways.

Results: Moxibustion exerted a significant analgesic effect for CIVP rats, mainly presenting as a decrease in the AWR score (all $\mathrm{P}<0.01$ ) under different levels of distending pressure and an increase in MWT and TWL thresholds (all $\mathrm{P}<0.05$ ). Compared with the normal group, 76 proteins were upregulated while 15 were downregulated, and MAPK signaling pathway was activated in the model group. Compared with the model group, there were 53 downregulated and 38 upregulated proteins in the moxibustion group, and MAPK signaling pathway was inhibited. Fold change (FC) $>1.3$ or $<0.77$ was taken as the screening standard to define the differentially expressed proteins. Fifteen differentially expressed proteins upregulated in the model group were downregulated in the moxibustion group. GO analysis showed that the differentially expressed proteins mainly controlled cellular metabolism regulation, transportation, and stress reactions. KEGG analysis revealed that these differentially expressed proteins were mostly involved in the ERK, JNK, and p38 pathways, and the ERK pathway was predominant.

Conclusion: Moxibustion mitigates CIVP in rats and inhibits the phosphorylation of proteins in the spinal MAPK signaling pathway. The analgesic effect of moxibustion may be associated with the regulation of the spinal MAPK signaling pathway.

Keywords: chronic inflammatory visceral pain, moxibustion, analgesia, spinal cord, MAPK signaling pathway

\section{Introduction}

Inflammatory bowel disease (IBD), including Crohn's disease (CD) and ulcerative colitis (UC), is an autoimmune disorder characterized by chronic nonspecific inflammation and damage in the intestinal lining. With changes in diet and the environment, the incidence of IBD has been rising on a global scale. ${ }^{1}$ Chronic inflammatory visceral pain (CIVP) is a major symptom in IBD, and mainly presents as recurrent and persistent abdominal pain. Visceral pain (VP) affects over $70 \%$ of all IBD patients, contributing to poor quality of life and loss of normal social 
interaction. Therefore, it is suggested that the priority for future IBD treatment should be the treatment of pain in combination with anti-inflammation measures. ${ }^{2}$ Currently, anti-inflammation is the top priority in the treatment of IBD, with the goal being the induction and maintenance of remission and repair of the intestinal mucosa, and VP is somehow overlooked. ${ }^{3}$

During recent years, there has been increasing use of moxibustion to treat IBD, and valid treatment efficacy and content treatment perception of patients with IBD have been observed. ${ }^{4-8}$ It has been shown that moxibustion can boost the repair of the intestinal lining, reduce inflammation in IBD patients, and ease CIVP (abdominal pain), though its mechanism is still unclear. ${ }^{9-11}$

Visceral hyperalgesia has been recognized as the most crucial mechanism in the development of CIVP, and central sensitization is the major pathological foundation for hyperalgesia and persistency of CIVP. ${ }^{12-14}$ The spinal cord is the initial relay station and integration center for pain signals that are transmitted to the brain center. It directly modulates pain information while receiving regulation signals from the center. Because of its key role in pain regulation, the spinal cord is fully involved in the research of central sensitization. ${ }^{15}$

The activation of the mitogen-activated protein kinase (MAPK) signaling pathway is involved in the onset and maintenance of visceral hypersensitivity. It promotes central sensitization in UC rat models via activating the expression of c-Fos in the central nervous system, so as to alter the visceral perception, emotions, and behaviors. ${ }^{16}$ Using inhibitors of key proteins of the MAPK signaling pathway not only benefits the repair of the colonic lining but also significantly mitigates hyperalgesia and paralgesia induced by nociceptive stimulations. Previous studies have indicated that the MAPK signaling pathway plays an important role in regulating the transmission and maintenance of pain signals. ${ }^{16-18}$ Our previous study revealed the noticeable analgesic effect of moxibustion for IBD-associated CIVP in rats, manifesting as the regulation of pain behaviors and pain emotions. ${ }^{19}$ However, additional evidence is required to prove whether this analgesic effect is related to the regulation of the MAPK signaling pathway in the spinal cord.

Therefore, this study prepared CIVP rat model to discuss the central mechanism of moxibustion analgesia in CIVP rats via observing the changes in the phosphorylated levels of different sites of MAPK signaling pathway in spinal cord of CIVP rats and the effect of moxibustion on the expressions.

\section{Materials And Methods}

\section{Experimental Animals}

Twenty-seven healthy male Sprague-Dawley (SD) rats weighing $150 \pm 20 \mathrm{~g}$ were purchased from Shanghai Super-B\&K Laboratory Animal Co., Ltd. (SCXK(Hu) 2013-0016). The rats received adaptive feeding for 1 week before the experiment began. All procedures in this experiment were in accordance with the Care and Use of Laboratory Animals issued by the Ministry of Science and Technology of the People's Republic of China ((2006)398) and were approved by the Animal Care and Use Committee of Shanghai University of Traditional Chinese Medicine (SZY 201605006). The 27 rats were randomized into a normal group (NG), a model group (MG), and a herb-partitioned moxibustion group (HPMG), with 9 rats in each group. The whole experimental schedule was described by a flowchart (Figure 1A).

\section{Main Reagents And Instruments}

The reagents and instruments procured for experiments were: moxa wool (Nanyang Hanyi Moxa, He'nan, China); aconite powder (Huaji Pharmaceutical Industry, Shanghai, China); MAPK (PMK185) protein array (Full Moon Biosystems, CA, USA); 2,4,6-trinitrobenzene sulfonic acid (TNBS, Sigma, MO, USA); p-cAMP response element-binding protein (CREB) primary antibody (Cell Signaling Technology, MA, USA); p-cJun primary antibody (Cell Signaling Technology, MA, USA); glyceraldehyde 3-phosphate dehydrogenase (GAPDH) primary antibody (Cell Signaling Technology, MA, USA); Von Frey filaments (Stoelting, IL, USA); BME2410A Thermal Stimulator (Institute of Medical Biology, Beijing, China); hematoxylin and eosin (HE) staining kit (Nanjing Jiancheng Technology, Nanjing, China); microarray scanner (Axon Instruments, CA, USA); GenePix Pro 6.0 (Axon Instruments, CA, USA); pathological analysis system (Leica, Wetzlar, Germany); light microscope and analysis system (Olympus, Tokyo, Japan); and Western blotting apparatus (Bio-Rad, CA, USA).

\section{Chronic Inflammatory Visceral Pain Modeling}

In this experiment, $5 \%(\mathrm{w} / \mathrm{v})$ TNBS and 50\% ethanol were mixed at 2:1 and enema was performed at $3 \mathrm{~mL} / \mathrm{kg}$ once a week for 4 consecutive weeks to prepare the CIVP rat model. ${ }^{20,21}$ After modeling, the rats all underwent pain behavior tests, and colonic histopathological observation with HE staining was performed for one rat from each group, to verify the success of the CIVP model. 
A

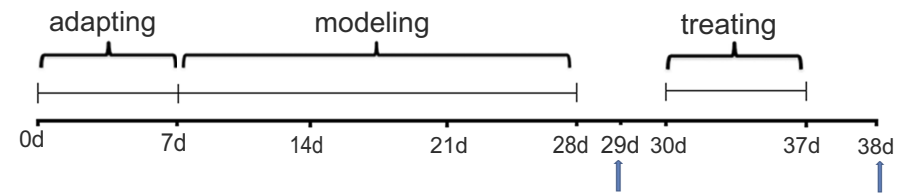

\section{H $\quad 0.01 .5160987$}

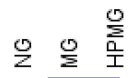

B
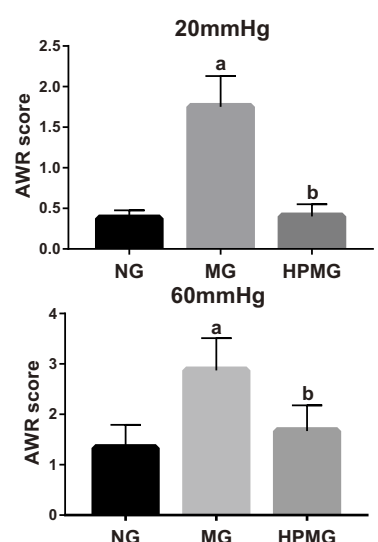

C

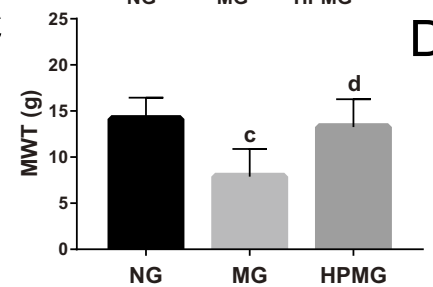

E

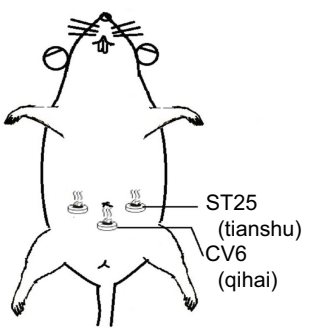

G

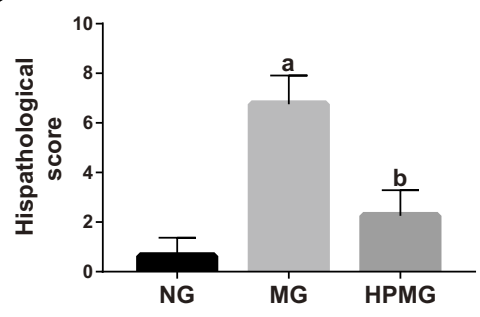

AWR/MWT/TWL

$40 \mathrm{mmHg}$

AWR/MWT/TWL
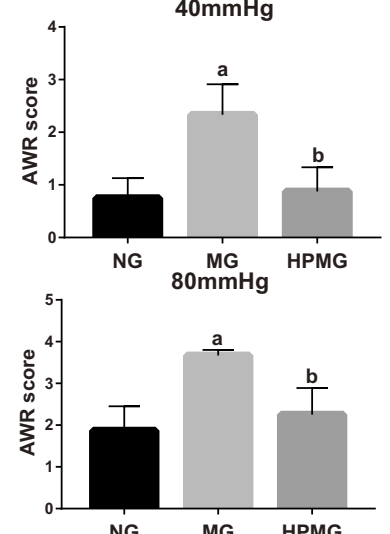

D

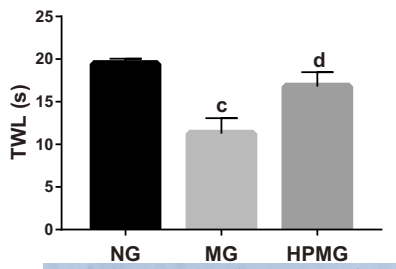

$\mathrm{F}$

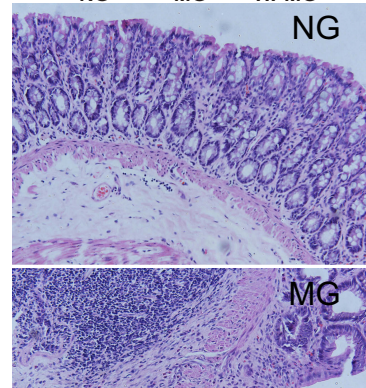

NG

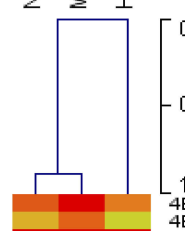

0.98135847

0.99067926

1.-BP1 Phospho-Thro

4E-BP1 (Phospho-Thr45)

ASK1 (Phospho-Sers3)

ATF2 (Phospo-Ser66)

ATF2 (Phospho-Ser62/44)

ATF2 (Phospho-Thr69/51)

ATF2 (Phospho-Thi7 1/53

ATF4 (Phospho- 245 )

c-Jun (Phospho-Se1243)

c-Jun (Phospho-Serb3)

c-Jun (Phospho-Ser73)

-Jun (Phosho-Thr239)

C-Jun (Phospho-Tyr170)

c-Kit (Phospho-Tyr721)

CREB (Phospho-Ser129)

IF2 alpha (Phospho-Ser51)

eIF4E (Phospho-Ser209)

Elk-1 (Phospho-Se1383)

Elk1 (Phospho-Ser389)

Estrogen Receptor-alpha (Phospho-Ser104)

(

Estrogen Receptor-alpha (Phospho-Ser118

Estrogen Receptor-alpha (Phospho-Ser167

FAK (Phospho-Tyr861)

Histone H2A.X (Phospho-Ser139)

Histone H3.1 (Phospho-Ser10)

HSF 1 (Phospho-Ser303)

HSP27 (Phospho-Ser15)

HSP27 (Phospho-Ser82

IRS-1 (Phospho-Ser307)

RS-1 (Phospho-Ser312)

RS-1 (Phospho-Ser636)

JunB (Phospho-Ser259)

JunB (Phospho-Ser79)

JunD (Phospho-Ser255)

MEF2A (Phospho-ThB12)

MEK1 (Phospho-Thr19)

MEK1 (Phospho-Sel221

MEK1 (Phospho-Thr291)

MEK-2 (Phospho-Thr394)

et (Phospho-Tyr1234)

MKK3 (Phospho-Ser189)

Myc (Phospho-Ser373)

Myc (Phospho-Thr358)

Myc (Phospho-Thr58)
P38 MAPK (Phospho-Tyr182)

P44/42 MAP Kinase (Phospho-Thr202)

(P2 MAP Kinase (Phospho-Thro2

53 (Phospho-Ser15)

553 (Phospho-Se1315)

p53 (Phospho-Ser33)

53 (Phospho-Ser46)

53 (Phospho-Ser6)

(Phospho-Ser9)

KC-delta (Phospho-

KC-theta (Phospho-Ser676)

Pyle (Phospho-Tyr402)

Raf1 (Phospho-Se1259)

SAPKJJNK (Phospho-Thr183)

APKIJNK (Phospho-Tyr183)

EK1/MKK4 (Phospho-Thr261)

Sre (Phospho-Tyr418)

sc (Phospho-Ty529)

athmin 1 (Phospho-Ser15)

Stathmin 1 (Phospho-Ser37)

Tau (Phospho-Se1214)

Tau (Phospho-Ser235)

Tau (Phospho-Ser356)

Tau (Phospho-Ser396)

Tau (Phospho-Ser404

Tau (Phospho-Ser422)

Tau (Phospho-Thr18

Tau (Phospho-Thr212)

Figure I Changes in rat pain behaviors, histopathological changes in the colon, and cluster of phosphorylated protein expression in the spinal cord MAPK signaling pathway. (A): Experimental schedule. (B): AWR scores under 20, 40, 60, $80 \mathrm{mmHg}$ CRD. (C): MWT; (D): TWL. (E): Illustration of herb-partitioned moxibustion. (F): Histopathological observations of the rat colon (HE staining, 200x). (G): Histopathological score. (H): Cluster of phosphorylated protein expression in the spinal cord MAPK signaling pathway (green denotes the average density, red represents a value higher than the average density, while blue indicates a value lower than the average density). $\mathrm{n}=8$ rats per group for panels $B, C, D$, and $G ; n=3$ rats per group for panel $H .{ }^{a} P<0.01$ vs $N G ;{ }^{c} P<0.05$ vs NG; ${ }^{b} P<0.01$ vs $M G$; ${ }^{d} P<0.05$ vs $M G$.

Abbreviations: AWR, abdominal withdrawal reflex; CRD, colorectal distension; MWT, mechanical withdrawal threshold; TWL, thermal withdrawal latency; NG, normal group; MG, model group; HPMG, herb-partitioned moxibustion group. 
Interventions were performed when the model was confirmed a success.

\section{Herb-Partitioned Moxibustion Intervention}

Rats in the HPMG were treated with herb-partitioned moxibustion (HPM) at Qihai (CV6) and bilateral Tianshu (ST25) (Figure 1E). A herbal cake was placed on the acupoints with the moxa cone on the top of it. The moxa cone was ignited, and burning proceeded until the cone was fully burnt. Two moxa cones were consecutively burnt (with each lasting for $10 \mathrm{~min}$ ) for each acupoint per treatment. HPM was performed once a day, for 7 times in total. Rats in the MG were not given any interventions except the same grasping and fixing operations as those that occurred in the HPMG. Rats in the NG did not receive modeling or intervention except the same grasping and fixing.

The moxa cones used in this study were made of fine moxa wools using the same mold. Each cone weighed $90 \mathrm{mg}$, and the dimensions were $0.4 \mathrm{~cm}$ in diameter and $0.4 \mathrm{~cm}$ in height. The herbal cakes were made of aconite powder using a mold $(0.6 \mathrm{~cm}$ in diameter and $0.3 \mathrm{~cm}$ in height), with yellow rice wine added prior to HPM treatment.

\section{Abdominal Withdrawal Reflex (AWR)}

AWR was used to estimate visceral hypersensitivity by referring to the Al-Chaer method. ${ }^{22}$ Four different levels of pressure, 20, 40,60, and $80 \mathrm{mmHg}$, were applied for colorectal distension. Each rat was tested 3 times, with each stimulation lasting for $20 \mathrm{~s}$ at an interval of $5 \mathrm{~min}$, and the average value of the three tests was considered as the final score.

\section{Mechanical Withdrawal Threshold (MWT)}

A Von Frey filament was used to perpendicularly stimulate the sole of a rat's hind paw, which lasted for less than $4 \mathrm{~s}$, and a positive reaction was defined by lifting or licking the paw. ${ }^{23}$ Each rat was tested 5 times consecutively.

\section{Thermal Withdrawal Latency (TWL)}

The TWL was measured using a thermal pain stimulator to radiate the rat's paw. The length of time from the beginning of the heat irradiation to the lifting of the paw was considered as the TWL. ${ }^{24}$ Each rat received five these tests at an interval of $3 \mathrm{~min}$. After the maximum and minimum values were removed, the mean value of the other three tests was defined as the final TWL. In this experiment, after intervention, the rats in each group all underwent the AWR, MWT, and TWL for behavioral observation.

\section{Sample Preparation}

Rats were euthanized via abdominal injection of pentobarbital sodium. Colons were collected and opened longitudinally. After rinsing with $4^{\circ} \mathrm{C}$ normal saline, the tissues were fixed in $10 \%$ neutral-buffered formalin solution. The spinal cord of $\mathrm{L}^{6}-\mathrm{S}^{2}$ was first placed in liquid nitrogen and then transferred to $\mathrm{a}-80^{\circ} \mathrm{C}$ freezer. Protein extraction was carried out within 1 month.

\section{Histopathological Observation Of The Colon}

Colon tissue slices were stained with HE and observed under a light microscope. HE procedure: After fixed in 10\% neutralbuffered formalin, colons were through dehydration, embedding, slicing and baking by using pathological analysis system, followed by dewaxing and dehydration by dimethylbenzene and graded ethanol, hematoxylin staining, differentiated by $1 \% \mathrm{HCl}$ and ethanol, blued by $1 \%$ ammonia solution, stained by $0.5 \%$ eosin, then again dehydrated through graded ethanol in order, transparentized by dimethylbenzene and sealed by neutral resin. Finally, the colon tissues were observed under light microscope and scored according to the standard: ulcer (none 0, small 1, mast 2); inflammation (none 0, light 1, serious 2); granuloma (none 0 , granuloma 1); lesion depth (none 0 , submucosa 1 , muscular layer 2 , serosa 3 ). ${ }^{25}$

\section{Total Protein Extraction From The Spinal Cord}

Each group contributed three spinal cord samples for total protein extraction. Lysis buffer was added at $1 \mathrm{~mL} / 250 \mathrm{mg}$ $(1 \mathrm{~mL}$ radioimmunoprecipitation assay (RIPA) buffer was mixed with $5 \mu \mathrm{L}$ protease inhibitor solution, $5 \mu \mathrm{L}$ phenylmethanesulfonyl fluoride (PMSF), and $5 \mu \mathrm{L}$ phosphatase/protease inhibitor cocktail), and homogenized at a low speed for a complete lysate of spinal cord tissues. The lysate was then centrifuged at $14000 \mathrm{rpm}$ for $15 \mathrm{~min}$. The supernatant was collected to detect the concentration of total protein by the bicinchoninic acid (BCA) method.

\section{Detection Of Phosphorylated Proteins Of The MAPK Signaling Pathway In The Spinal Cord}

This process was performed by strictly following the instructions of the Full Moon protein array kit. For the assay, $50 \mu \mathrm{l}$ 
reaction mixture containing $100 \mu \mathrm{g}$ total protein was labeled with $10 \mu \mathrm{L}$ biotin/ $N, N$-dimethylformamide (DMF). After reaction for $30 \mathrm{~min}$ at room temperature, the array was rinsed with Milli-Q water for $3 \mathrm{~min}$, and then, the reaction was terminated by adding $30 \mu \mathrm{L}$ stop solution. The chip's surface was fully soaked in $6 \mathrm{~mL}$ Protein Coupling Mix, and the coupling chamber was then covered and maintained overnight at $4^{\circ} \mathrm{C}$. It was then washed twice with Milli-Q water. Afterwards, the chips were incubated in Cy3-streptavidin solution at room temperature. After the reaction mixtures were removed, the chips were dried and scanned.

\section{Image Scanning And Data Analysis}

Images and primary data were acquired with the Axon GenePix scanner and GenePix Pro 6.0 software. Grubbs' criterion was used to process the primary data. The mean fluorescence intensity value, standard deviation (SD), and coefficient of variation $(\mathrm{CV})$ at each protein site were calculated. The phosphorylation levels of the 91 sites on the antibody were analyzed by the ratio between the phosphorylated value and unphosphorylated value. Fold change $(\mathrm{FC})>1.3$ or $<0.77$ was taken as the screening standard to define the differentially expressed proteins in the MAPK signaling pathway in the rat spinal cord between different groups.

\section{Gene Ontology (GO) And Kyoto Encyclopedia Of Genes And Genomes (KEGG) Analyses}

The corresponding UniProt accession of the differentially expressed proteins was searched in the UniProt Knowledgebase (UniProt KB) database for analysis of structure and physiochemical features. GO and KEGG were retrieved, and the target proteins underwent functional cluster and pathway analysis using OmicsBean software.

\section{Validation Of Differentially Expressed Proteins In The Spinal Cord}

Western blotting (WB) was used to determine the differentially expressed proteins. The total protein in the spinal cord was extracted, and the amount was determined using the BCA method. The $50 \mu \mathrm{g}$ of total protein was processed by electrophoresis at $100 \mathrm{~V}$ for $2 \mathrm{~h}$, and then underwent wet membrane transfer at $350 \mathrm{~mA}$ for $1 \mathrm{~h}$. The membrane was incubated with the primary antibody $(1: 1000)$ at $4^{\circ} \mathrm{C}$ overnight, horseradish peroxidase (HRP)-labeled secondary antibody (1:1000) for 2 $\mathrm{h}$, and then with ECL luminescence reagent for $2 \mathrm{~min}$. A BioRad gel imaging system was used for automatic imaging. The grey values of target protein bands and internal reference were determined, and their ratio was taken as the relative expression level of the target protein.

\section{Statistical Analysis}

SPSS version 19.0 (IBM, Armonk, NY, USA) software was used for the data analysis. The data were first tested for normal distribution and homogeneity of variance. The measurement data that conformed to normal distribution were expressed as the mean $\pm \mathrm{SD}$; if not, the data were expressed as median (P25, P75). For the data in accordance with normal distribution and homogeneity of variance, one-way Analysis of Variance (ANOVA) analysis was applied for inter-group comparison and least significant difference (LSD) for paired comparison; the Games-Howell method was used for intergroup comparison if there was heterogeneity of variance. When the data did not conform to normal distribution, a non-parametric test was employed. $\mathrm{P}<0.05$ was considered to indicate statistical significance.

\section{Results}

\section{AWR Score (AWR)}

Compared with the NG, the AWR scores in the MG significantly increased under the four different distending pressures $(20,40,60$, and $80 \mathrm{mmHg}$ ) (all $\mathrm{P}<0.01$ ). Compared with the MG, AWR scores in the HPMG significantly decreased under each distending pressure (all $\mathrm{P}<0.01$ ) (Figure 1B).

\section{MWT And TWL}

Compared with the NG, MWT and TWL in the MG significantly decreased (all $\mathrm{P}<0.05$ ). Compared with the $\mathrm{MG}$, MWT and TWL in the HPMG significantly increased (all $\mathrm{P}<0.05$ ). (Figure $1 \mathrm{C}$ and $\mathrm{D}$ ).

\section{Morphological Changes In The Colon}

In the NG, the colons of the rats had a clear structure, with complete lining, aligned glands, and without inflammation, congestion, or edema. In the $\mathrm{MG}$, the colon tissues were damaged, with incomplete lining, ulcers, disordered gland arrangement, significant inflammatory infiltration in the mucosa and submucosa of predominantly neutrophils and eosinophils, and significant congestion and edema in connective tissues. Compared to $\mathrm{NG}$, the histopathological scores of rats in MG were increased significantly $(\mathrm{P}<0.01)$. Colons in the HPMG were substantially complete in structure, with successive 
lining, without notable ulcers, with certain gland hyperplasia but with normal arrangement, although slight inflammatory infiltration in the mucosa and submucosa, and mild-moderate swelling in connective tissues were observed (Figure 1F). Compared to MG, the histopathological scores of rats in HPMG were reduced obviously $(\mathrm{P}<0.01)$. (Figure $1 \mathrm{G})$

\section{Phosphorylation Level Of Proteins In The Spinal MAPK Signaling Pathway}

Compared with the NG, the phosphorylation levels of 76 proteins were upregulated in the spinal cord in the $\mathrm{MG}$, among which, 33 had FC $>1.3$ (Table 1, Figure 1H); 15 proteins were downregulated, of which, two had $\mathrm{FC}<0.77$ (Table 2, Figure 1H).

Compared with the MG, the phosphorylation levels of 53 proteins in the spinal cord in the HPMG were downregulated, and 25 had $\mathrm{FC}<0.77$ (Table 3, Figure 1H); 38 proteins were upregulated, and eight showed $\mathrm{FC}>1.3$ (Table 4, Figure 1H).

\section{GO And KEGG Analyses Of The Differentially Expressed Proteins}

As mentioned above, $\mathrm{FC}>1.3$ or $<0.77$ was considered as the standard to determine the differentially expressed proteins. ${ }^{26-28}$ Compared with the NG, 33 sites of 21 proteins were upregulated in the spinal cord MAPK signaling pathway, and these proteins mainly control cell movement regulation, positive control over biological response, and cell stress reaction modulation (Supplementary Table 46, Figure 2A). There were nine signaling pathways that were extensively involved, predominated by MAPK and phosphatidylinositol 3-kinase (PI3K) pathways (Figure 2B). Two sites from the same protein showed downregulated phosphorylation levels and were not selected for GO and KEGG analyses.

Compared with the MG, 25 sites from 19 proteins in the spinal MAPK signaling pathway were downregulated in the HPMG. These proteins mainly function to regulate stress reactions and positively regulate biological processes (Supplementary Table 57-1,2, Figure 3A). Eight signaling pathways were involved, concentrating on MAPK and PI3K pathways (Figure 3B). Eight sites from seven proteins were upregulated, and these proteins regulate stress reactions, and positively control genetic expression and cellular metabolism. (Figure 4A). Two signaling pathways were involved and were predominated by the MAPK signaling pathway (Figure 4B).
Table I Upregulated Phosphorylated Proteins And Sites In the MAPK Signaling Pathway Associated With CIVP Pathogenesis

\begin{tabular}{|l|l|l|}
\hline Protein (Phosphorylated Site) & FC (MG/NG) & P value \\
\hline 4E-BPI (Phospho-Thr36) & 1.46 & 0.14 \\
4E-BPI (Phospho-Thr45) & 1.41 & 0.21 \\
ASKI (Phospho-Ser83) & 1.57 & 0.13 \\
ATF2 (Phospho-SerII2/94) & 1.31 & 0.18 \\
c-Jun (Phospho-Ser73) & 1.83 & 0.15 \\
c-Jun (Phospho-Thr239) & 1.37 & 0.23 \\
CREB (Phospho-Ser I29) & 1.38 & 0.08 \\
elF2alpha (Phospho-Ser5I) & 1.66 & 0.11 \\
elF4E (Phospho-Ser209) & 1.35 & 0.13 \\
ElkI (Phospho-Ser389) & 1.41 & 0.06 \\
Estrogen Receptor-alpha (Phospho-Ser 106) & 1.56 & 0.06 \\
Estrogen Receptor-alpha (Phospho-SerI67) & 1.41 & 0.09 \\
FAK (Phospho-Tyr86I) & 1.32 & 0.26 \\
FAK (Phospho-Tyr925) & 1.31 & 0.11 \\
Histone H3.I (Phospho-Ser I0) & 1.94 & 0.15 \\
HSFI (Phospho-Ser303) & 1.4 & 0.21 \\
IRS-I (Phospho-Ser307) & 1.47 & 0.1 \\
IRS-I (Phospho-Ser636) & 1.46 & 0.12 \\
IRS-I (Phospho-Ser639) & 1.44 & 0.13 \\
JunB (Phospho-Ser259) & 1.36 & 0.15 \\
JunB (Phospho-Ser79) & 1.53 & 0.15 \\
Met (Phospho-Tyr 1234) & 1.45 & 0.17 \\
Myc (Phospho-Ser373) & 1.37 & 0.08 \\
Myc (Phospho-Thr358) & 1.31 & 0.15 \\
PKC-delta (Phospho-Ser645) & 2.22 & 0 \\
PKC-theta (Phospho-Ser676) & 1.41 & 0.19 \\
Pyk2 (Phospho-Tyr402) & 1.61 & 0.18 \\
StathminI (Phospho-SerI5) & 1.45 & 0.14 \\
StathminI (Phospho-Ser24) & 0.14 \\
Tau (Phospho-Ser2I4) & 0.22 \\
Tau (Phospho-Ser235) & 0.15 \\
Tau (Phospho-Ser396) & 1.37 \\
Tau (Phospho-Thr23I) & 0.09 \\
\hline
\end{tabular}

Abbreviations: FC, fold change; NG, normal group; MG, model group.

Table 2 Downregulated Phosphorylated Proteins And Sites In The MAPK Signaling Pathway Associated With CIVP Pathogenesis

\begin{tabular}{|l|l|l|}
\hline Protein (Phosphorylated Site) & FC (MG/NG) & P value \\
\hline Tau (Phospho-Thr2I2) & 0.76 & 0.23 \\
Tau (Phospho-Ser422) & 0.75 & 0.29 \\
\hline
\end{tabular}

Abbreviations: FC, fold change; NG, normal group; MG, model group.

\section{Screening Of The Differentially Expressed Proteins}

Comprehensive analysis revealed 15 protein sites that were upregulated in the MG (compared with the NG) and were downregulated in the HPMG (compared with the MG) (Table 5). There was no site downregulated in 
Table 3 Downregulated Phosphorylated Proteins And Sites In The MAPK Signaling Pathway Associated With Moxibustion Treatment Of CIVP

\begin{tabular}{|l|l|l|}
\hline Protein (Phosphorylated Site) & FC (HPMG/MG) & P value \\
\hline 4E-BPI (Phospho-Thr36) & 0.76 & 0.11 \\
4E-BPI (Phospho-Thr45) & 0.77 & 0.27 \\
ASKI (Phospho-Ser966) & 0.66 & 0.25 \\
c-Jun (Phospho-Ser73) & 0.65 & 0.14 \\
c-Kit (Phospho-Tyr72I) & 0.62 & 0.04 \\
CREB (Phospho-Ser I29) & 0.68 & 0.43 \\
elF2 alpha (Phospho-Ser5I) & 0.7 & 0.49 \\
elF4E (Phospho-Ser209) & 0.76 & 0.23 \\
Histone H2A.X (Phospho-Ser I39) & 0.72 & 0.3 \\
IRS-I (Phospho-Ser307) & 0.59 & 0.49 \\
IRS-I (Phospho-Ser636) & 0.69 & 0.2 \\
IRS-I (Phospho-Ser639) & 0.6 & 0.17 \\
JunB (Phospho-Ser259) & 0.7 & 0.46 \\
MEKI (Phospho-Thr29I) & 0.55 & 0.12 \\
Met (Phospho-Tyr 234) & 0.72 & 0.16 \\
Met (Phospho-Tyr 1349) & 0.57 & 0.17 \\
P44/42 MAPKinase (Phospho-Thr202) & 0.69 & 0.36 \\
P53 (Phospho-Ser6) & 0.6 & 0.03 \\
PKC-delta (Phospho-Ser645) & 0.56 & 0.17 \\
PKC-theta (Phospho-Ser676) & 0.58 & 0.05 \\
Pyk2 (Phospho-Tyr402) & 0.75 & 0.2 \\
StathminI (Phospho-Ser I5) & 0.74 & 0.13 \\
StathminI (Phospho-Ser24) & 0.72 & 0.11 \\
Tau (Phospho-Ser422) & 0.59 & 0.06 \\
Tau (Phospho-Thr2I2) & 0.75 & \\
\hline
\end{tabular}

Abbreviations: FC, fold change; MG, model group; HPMG, herb-partitioned moxibustion group.

Table 4 Upregulated Phosphorylated Proteins And Sites In The MAPK Signaling Pathway Associated With Moxibustion Treatment Of CIVP

\begin{tabular}{|l|l|l|}
\hline Protein (Phosphorylated Site) & FC(HPMG/MG) & P value \\
\hline ASKI (Phospho-Ser83) & 1.33 & 0.21 \\
Elk-I (Phospho-Ser383) & 1.6 & 0.25 \\
Histone H3.I (Phospho-Ser 10) & 1.33 & 0.13 \\
MEK-2 (Phospho-Thr394) & 1.76 & 0.44 \\
Myc (Phospho-Ser373) & 1.33 & 0.11 \\
P38 MAPK (Phospho-Tyr 182) & 1.41 & 0.32 \\
P53 (Phospho-Ser37) & 1.34 & 0.23 \\
P53 (Phospho-Ser46) & 1.52 & 0.43 \\
\hline
\end{tabular}

Abbreviations: FC, fold change; MG, model group; HPMG, herb-partitioned moxibustion group.

the MG (compared with the NG) that was upregulated in the HPMG (compared with the MG). Among the 15 differential proteins, eight were from the MAPK-extracellular regulated protein kinases (ERK) signaling pathway, four from the MAPK/c-Jun N-terminal kinase (JNK) signaling pathway, and three from the MAPK/p38 signaling pathway. These data indicate that moxibustion can simultaneously regulate the three pathways, and MAPK/ERK may play a crucial role in the process of moxibustion analgesia.

\section{Verification Of Differential Proteins $\mathrm{p}$-CREB And $\mathrm{p}$-cJun}

Differential proteins $\mathrm{p}-\mathrm{CREB}$ and $\mathrm{p}$-cJun were selected for verification. Compared with the NG, the protein expression of p-CREB and p-cJun in the spinal cord was significantly higher in the $\mathrm{MG}$ (both $\mathrm{P}<0.01$ ). Compared with the MG, the protein expression of p-CREB and p-cJun in the rat spinal cord was significantly decreased in the HPMG $(\mathrm{P}<0.01, \mathrm{P}<0.05)$ (Figure 5).

\section{Discussion}

Thus far, clinical management of chronic visceral pain (CVP) has rarely been reported. Some studies mentioned the use of tricyclic antidepressant (TCA), selective serotonin reuptake inhibitor (SSRI), and serotonin and noradrenaline reuptake inhibitor (SNRI) to treat CVP based upon various analgesics. ${ }^{29}$ However, there is still a lack of reliable evidence of the benefits of opioids for chronic non-cancerous pain in either adults or children, and additionally, their use may be detrimental, as exclusive use of central analgesics may incur opioid-induced hyperalgesia (e.g., narcotic bowel syndrome). ${ }^{12,30}$ As one type of CVP, CIVP is pathologically featured by recurrent inflammatory injury and is commonly seen in IBD. It greatly affects the quality of life for patients and undermines their social and work abilities.

It is yet unclear what causes CIVP, but it is suggested that peripheral factors (intestinal inflammation, dysfunction, visceral hypersensitivity), and neurological and psychological factors mediated by the central nervous system participate in the development of CIVP, ${ }^{12}$ which makes it difficult to treat. Treatment is usually initiated with antiinflammation agents, as there are no specific treatment methods or drugs for this medical condition. In recent years, consensus has been reached that sensitization of both peripheral and central nerves is involved in the pathogenesis of CIVP, and single-target treatment thus may have certain limitations. ${ }^{31}$ It is a multi-target and multichannel process when acupuncture and moxibustion stimulate acupoints. Therefore, acupuncture and moxibustion bear a certain advantage in treating CIVP. ${ }^{32,33}$ 
A

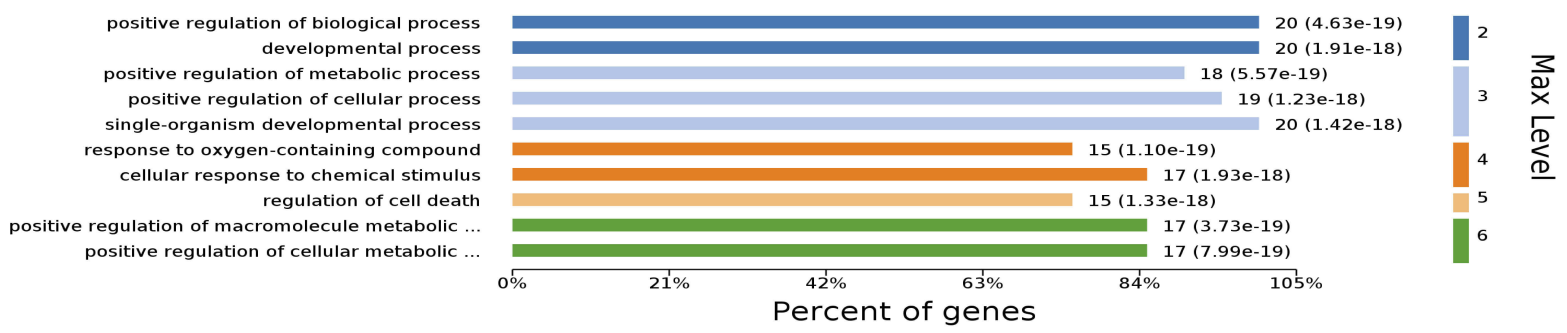

B

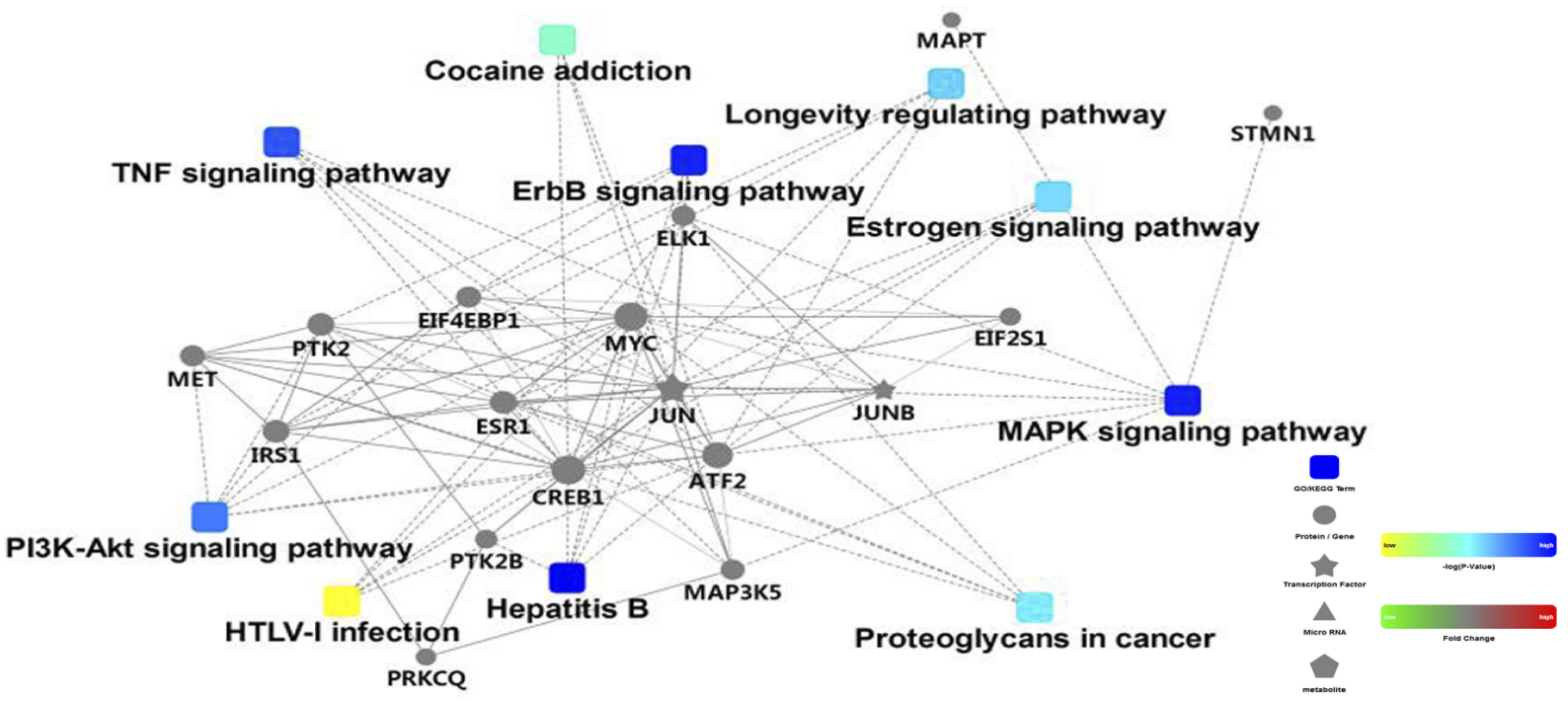

Figure 2 Differential proteins associated with CIVP pathogenesis based on the MAPK signaling pathway (MG vs NG). (A): Protein functional cluster. (B): Corresponding signaling pathways and protein interactions. $\square$ denotes a GO/KEGG term; $\circ$ represents protein/gene; is denotes a transcription factor; blue color shows a high -log (Pvalue), and yellow color shows a low -log (P-value); red color denotes a high fold change, and green color indicates a low fold change.

With progressive research, there is now a sufficient body of evidence indicating that analgesia via acupuncture is effective, but not everyone can tolerate its mechanical stimulation. ${ }^{34,35}$ Compared to acupuncture, moxibustion is mild and comfortable, and thus more easily accepted. Moxibustion has been proved effective for intestinal diseases accompanied by abdominal pain and diarrhea. For example, Zhao et al found that moxibustion produced some effects in improving symptoms in constipation-predominant irritable bowel syndrome (C-IBS) such as abdominal pain, bloating, and mental symptoms, probably through modulating of the brain-gut axis function. ${ }^{36}$ Bao et al indicated that both electroacupuncture and moxibustion improved cortex-subcortical coupling in remissive CD patients, but the former regulated homeostatic afferent processing network, while moxibustion mainly regulated the default mode network of the brain. ${ }^{37}$ These evidences show that moxibustion is effective in alleviating the visceral pain. The current study observed how moxibustion affected CIVP in a rat model. We found that moxibustion significantly downregulated the AWR score $(\mathrm{P}<0.01)$ and upregulated the MWT and TWL $(\mathrm{P}<0.05)$ in CIVP rats, suggesting a satisfactory analgesic effect, which agreed with previous studies. ${ }^{19,38,39}$ Although moxibustion is characterized by valid efficacy, convenient operation, and mild stimulation, and has been gradually adopted to treat IBD and CIVP, its action mechanism is vague.

The MAPK signaling pathway adjusts neuron plasticity and the central sensitization of pain, which induces and maintains hyperalgesia. Activation of this pathway is involved in visceral pain and associated pain behaviors in DSS (Dextran Sulfate Sodium Salt)-induced UC. ${ }^{16,40,41}$ MAPK inhibitors extremely influence pain sensation under normal conditions but significantly ease hyperalgesia after tissue injuries, which implies that regulating the MAPK signaling pathway may be the 

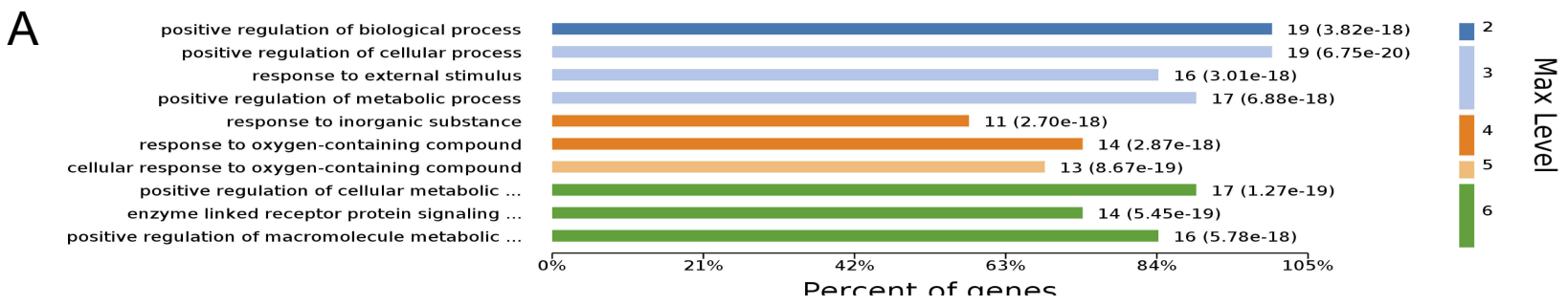

B

Acute myeloid leukemia

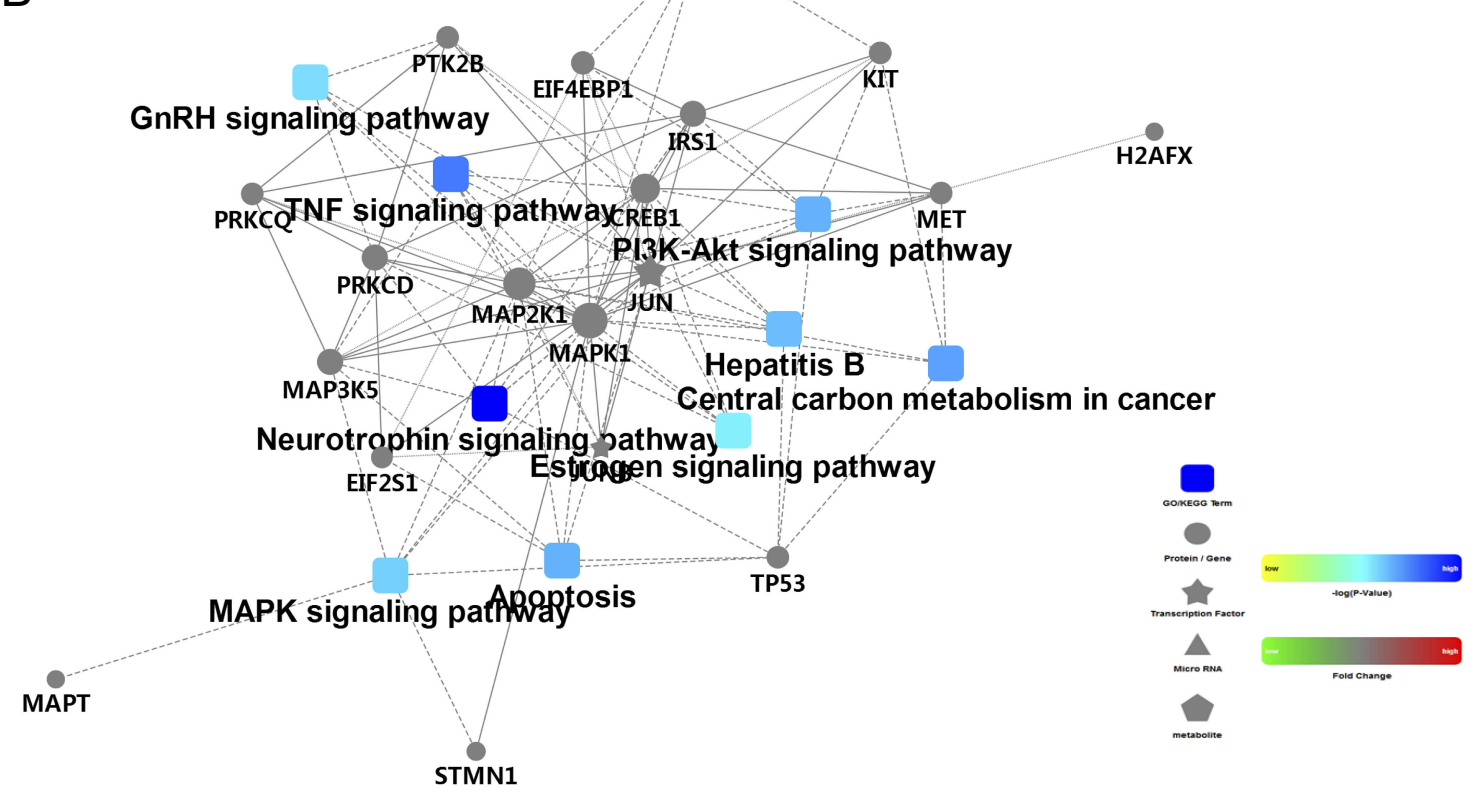

Figure 3 Downregulated differential proteins in the MAPK signaling pathway associated with moxibustion treatment of CIVP (HPMG vs MG). (A): Protein functional cluster. (B): Corresponding signaling pathways and protein interactions. $\square$ denotes a GO/KEGG term; $\circ$ represents protein/gene; $\downarrow$ denotes a transcription factor; blue color shows a high -log (P-value), and yellow color shows a low -log (P-value); red color denotes a high fold change, and green color indicates a low fold change.

mechanism in treating hyperalgesia after tissue injuries. ${ }^{42}$ The MAPK family consists of four subgroups: ERK, p38MAPK, JNK/SAPK (stress-activated protein kinase), and ERK5. ERK, p38MAPK, and JNK are all associated with pain. ${ }^{43-45}$ The MAPK signaling pathway is closely related to IBD. The activation of ERK and JNK mediates pain signals in acute UC, and they both participate in the analgesic effect of morphine. ${ }^{46,47}$ Therefore, the systemic study of MAPKs can help further elucidate the development of CIVP and provide novel intervention targets for the management of CIVP.

In this study, we focused on the changes in the protein expression profile of the MAPK signaling pathway in the rat spinal cord using a high-throughput phosphorylated protein microarray. The protein microarray analysis in this study found, compared with the normal group, 76 (76/91) sites showed up-regulated phosphorylated level in the spinal cord MAPK signaling pathway in CIVP rats and the phosphorylated level of MAPK signaling pathway in spinal cord presented an increasing tendency. Taking FC $>1.3$ as the screening standard, 33 differentiated protein sites were activated in the spinal cord of CIVP rats, and the activation might be involved in the development of CIVP. The functional cluster analysis by GO analysis showed that these protein sites are mainly involved in the positive regulation of biological process, development process, positive regulation of metabolic process, positive regulation of cellular process and single-organism development process. Compared with the model group, the herb-partitioned moxibustion group had 53 (53/91) protein sites showing a down-regulated phosphorylated level of MAPK signaling pathway in the spinal cord, indicating that the inhibition of these 25 differentiated protein sites might be critical mechanism of herb-partitioned 


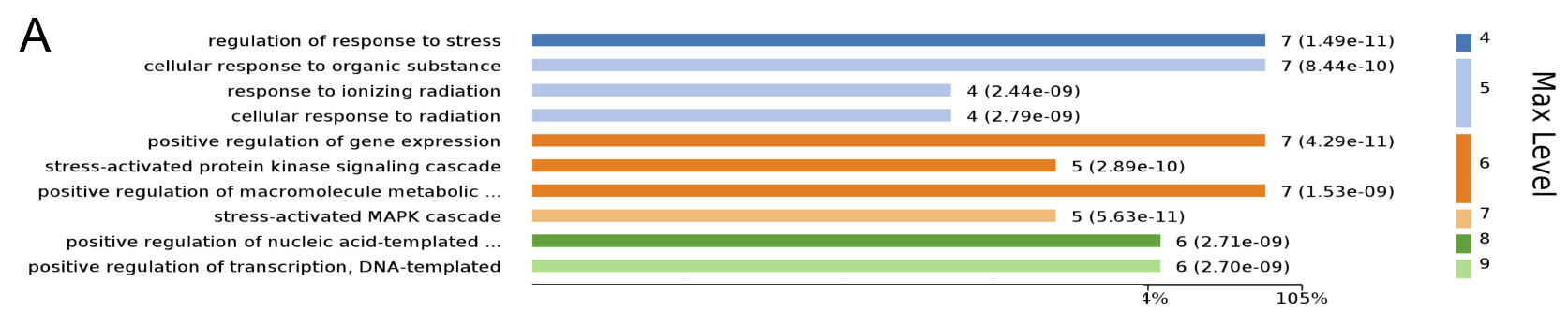

B

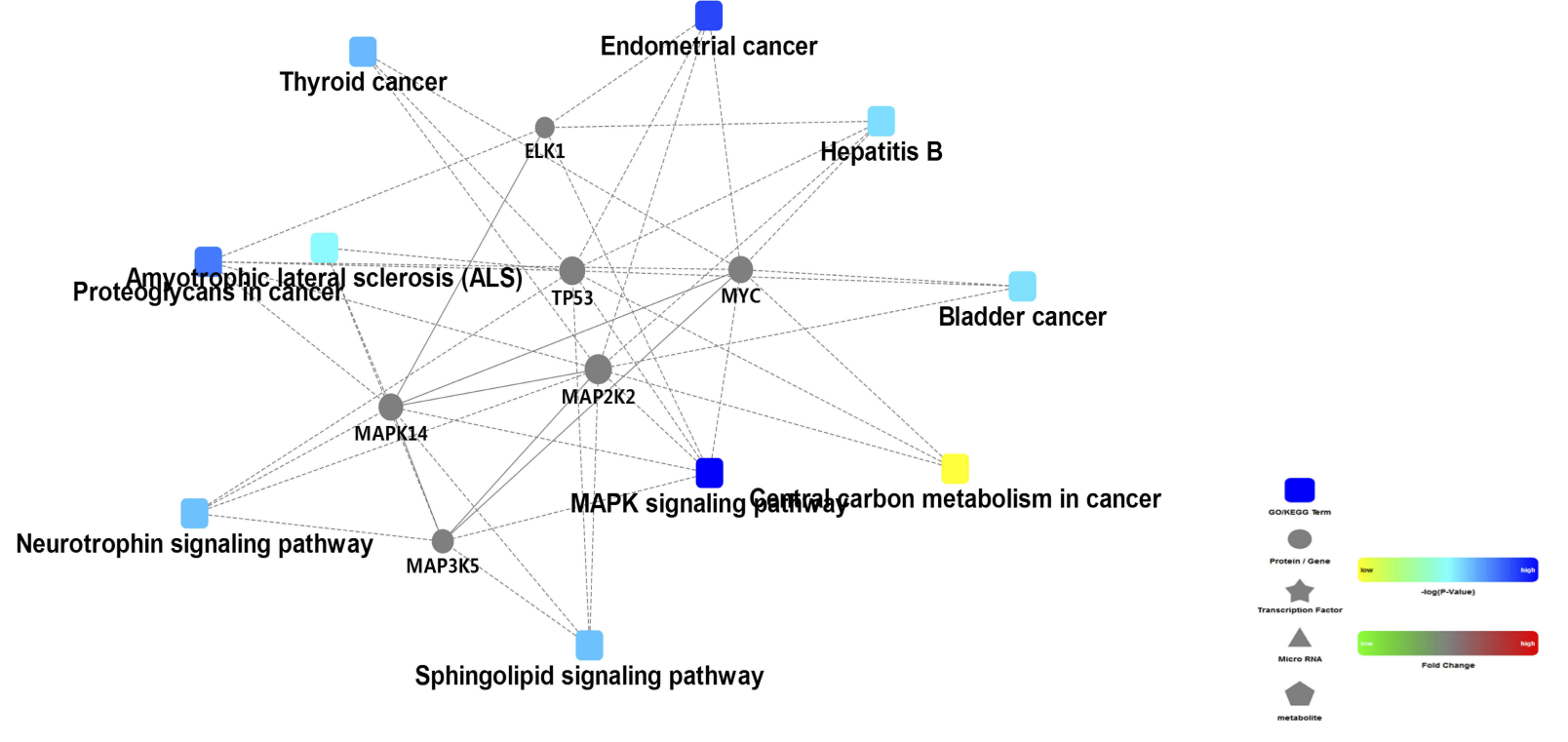

Figure 4 Upregulated differential proteins in MAPK signaling pathway associated with moxibustion treatment of CIVP (HPMG vs MG). (A): Protein functional cluster. (B): Corresponding signaling pathways and protein interactions. $\square$ denotes a GO/KEGG term; $\circ$ represents protein/gene; $i$ denotes a transcription factor; blue color shows a high - log (P-value), and yellow color shows a low -log ( $\mathrm{P}$-value); red color denotes a high fold change, and green color indicates a low fold change.

Table 5 List Of 15 Differentially Expressed Proteins

\begin{tabular}{|l|l|l|}
\hline Protein (Phosphorylated Site) & FC (MG/NG) & FC (HPMG/MG) \\
\hline 4E-BPI (Phospho-Thr36) & 1.46 & 0.76 \\
4E-BPI (Phospho-Thr45) & 1.41 & 0.77 \\
C-Jun (Phospho-Ser73) & 1.83 & 0.65 \\
CREB (Phospho-Ser 129) & 1.38 & 0.68 \\
elF4E (Phospho-Ser209) & 1.35 & 0.76 \\
IRS-I (Phospho-Ser307) & 1.47 & 0.59 \\
IRS-I (Phospho-Ser636) & 1.46 & 0.69 \\
IRS-I (Phospho-Ser639) & 1.44 & 0.6 \\
JunB (Phospho-Ser259) & 1.36 & 0.7 \\
Met (Phospho-TyrI234) & 1.45 & 0.72 \\
PKC-delta (Phospho-Ser645) & 2.22 & 0.55 \\
PKC-theta (Phospho-Ser676) & 1.41 & 0.58 \\
Pyk2 (Phospho-Tyr402) & 1.61 & 0.75 \\
StathminI (Phospho-Ser 15) & 1.45 & 0.74 \\
StathminI (Phospho-Ser24) & 1.42 & 0.72 \\
\hline
\end{tabular}

Abbreviations: FC, fold change; NG, normal group; MG, model group; HPMG, herb-partitioned moxibustion group.
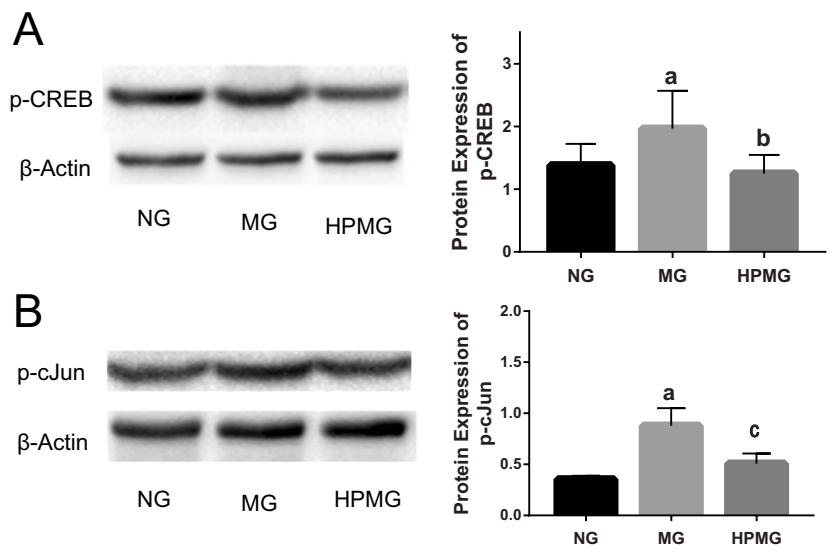

Figure 5 Phosphorylated protein expression of CREB and cJun in the spinal cord. (A): Phosphorylated protein expression of CREB in the spinal cord; (B): phosphorylated protein expression of cJun in the spinal cord. $n=8$ rats per group. ${ }^{a} P<0.01$ vs $N G$; ${ }^{b} P<0.01$ vs $M G$; ${ }^{c} P<0.05$ vs $M G$.

Abbreviations: NG, normal group; MG, model group; HPMG, herb-partitioned moxibustion group. 
moxibustion producing treatment efficacy and analgesic effect for CIVP. GO analysis revealed that this group of protein sites are mainly involved in enzyme binding, protein binding, protein kinase binding, phosphatase binding, kinase binding, etc. These results showed that herb-partitioned moxibustion not only targets the key activation proteins of the signaling pathway but also the binding of proteins in treatment of CIVP. Meanwhile, these differentially expressed proteins mostly work for the positive regulation of biological process, positive regulation of cellular process, response to external stimulus, positive regulation of metabolic process, response to inorganic substance, etc. The function of the differentially expressed proteins in the model group and in the herb-partitioned moxibustion group overlapped significantly, suggesting that the mechanism of herb-separated moxibustion in treating CIVP may be characterized by multi-target, multifunction, multi-level and multi-pathway.

The mechanism of moxibustion analgesia has not been adequately explained thus far. Some studies only support the effect of moxibustion in relieving functional visceral pain but cannot ensure its effect for other forms of pain. ${ }^{36,48,49}$ Zhu et al found that the analgesic effect that moxibustion produced for functional visceral pain should be associated with the regulation of the prefrontal cortex (PFC) and anterior cingulate cortex (ACC), indicating a central regulation mechanism of moxibustion analgesia. ${ }^{50}$ The effect of moxibustion (including analgesia) is mainly generated through the stimulation of acupoints by the thermal effects, radiation effects, and pharmacological actions of moxa and its combustion products, which is mainly characterized by thermal stimulation. ${ }^{51}$ The study of its effect mechanism is mainly started with warm-heat stimulation of acupoints. It is found that the transient receptor potential vanilloid (TRP) and purine receptor ( $\mathrm{P} 2$ receptor) play a vital role in the initiation of moxibustion effect and participate in the regulation of pain and inflammatory response. This regulatory effect occurs in every part of human body's "neuro-endocrine-immune" network, and affects many analgesic/painmediated factors and inflammatory cytokines. ${ }^{52-56}$ The mechanism of pain formation and development is quite complicated, and many neural active substances are involved in the central and peripheral regulation of pain. The balance of analgesic/anti-analgesic system is the ultimate manifestation of pain relief. And the suppression of uplink and regulation of downlink pain-related signals may be one of the important ways of analgesic effects for moxibustion. The spinal cord is the lower center of pain signal processing. In this study, we observe the regulatory effect of moxibustion on the phosphorylation level of MAPK signaling protein at different sites in the spinal cord of CIVP rats, screen the key molecules and related signaling pathways of moxibustion analgesia, and explore the central mechanism of moxibustion analgesic effect on CIVP rats at the spinal cord level. The present study found that moxibustion successfully mitigated CIVP and inhibited the phosphorylation level of proteins, especially c-CREB and p-cJun, in the MAPK signaling pathway in the rat spinal cord in a CIVP model. It is suggested that moxibustion may relieve visceral pain in CIVP rats through regulating the activation of neurons in the spinal cord (central sensitization). Our previous studies also showed that moxibustion downregulated the phosphorylated protein expression of MEK1 (mitogen-activated extracellular signal-regulated kinase 1), ERK1/2, CREB, and ERK in the spinal cord of CIVP rats, again proving that inhibiting the MAPK signaling pathway, i.e., the MEK/ERK/ CREB signaling pathway, in the spinal cord should be one of the central mechanisms in moxibustion analgesia. The question subsequently arises as to whether other paths of the MAPK signaling pathway also participate in the process.

To take a deeper look, we examined the changes in the phosphorylated protein levels in the spinal MAPK signaling pathway using the 91-proteins microarray technique, to screen the pathways of MAPK that were possibly involved in moxibustion analgesia. We showed that moxibustion actually affected three pathways in MAPK (MAPK/ERK, MAPK/JNK, and MAPK/p38), of which, the MAPK/ERK pathway contributed eight differentially expressed proteins, and thus was considered as the main route of the MAPK signaling pathway in moxibustion analgesia, which also agreed with previous studies. ${ }^{38,39}$ However, further exploration is required for the other two pathways, MAPK $/ J N K$ and MAPK/p38. At the same time, although the microarray detection mainly focused on the important signal proteins of MAPK signaling pathway, the screening results, together with KEGG analysis, showed that the occurrence of IBD-related CIVP may also be associated with PI3K-Akt signaling pathway, hepatitis B signaling pathway, TNF signaling pathway, and ErbB signaling pathway; the analgesic effect of herb-partitioned moxibustion may also be related to the modulation of PI3K-Akt signaling pathway, neurotrophin signaling pathway, TNF signaling pathwayand apoptosis signaling pathway. However, the verification requires further studies.

\section{Conclusion}

Moxibustion can mitigate visceral hyperalgesia in CIVP rat models, inhibit the phosphorylated protein levels of the 
MAPK signaling pathway in the spinal cord, and regulate three pathways (MAPK/ERK, MAPK/JNK, MAPK/p38) in MAPK, of which, MAPK/ERK may be the main MAPK signaling pathway involved in the process of moxibustion analgesia.

\section{Prospects}

Because moxibustion can produce a multi-path, multilevel, and multi-target effect, moxibustion analgesia is a complicated process. Therefore, it may take a long time to completely understand how it works. This study simply used the MAPK signaling pathway in the spinal cord as the target to analyze the possible mechanism of moxibustion treatment of CIVP, which is a rather limited scale. CIVP is complex in its development and persistence, and involves the interactions of various pathways and transmitters. Although our results only suggest a notable regulation of moxibustion on the MAPK/ERK, JNK, and p38 pathways, we could not assure that the ERK5 signaling pathway was not involved, despite its minimal contribution in protein screening. Moreover, the KEGG analysis showed that the MAPK signaling pathway was not the only one involved in the development of CIVP and moxibustion analgesia, as the PI3K signaling pathway largely participated. Therefore, whether they work jointly or separately can be another research target, which may help thoroughly unveil the analgesia mechanism of moxibustion for CIVP and provide reliable evidence to explain moxibustion analgesia.

\section{Acknowledgements}

This study was supported by the National Natural Science Foundation of China (No. 81273843, 81674073, 81202754); Training Project for Outstanding Discipline Leaders of Shanghai Municipal Commission of Health and Family Planning (No. 2017BR047); National Key Basic Research Program of China (973 Program) (No. 2015CB554501); Budgetary Project of Shanghai University of Traditional Chinese Medicine (No.18LK050). Microarray experiments in this study were performed by Wayen Bio-tech, Shanghai, China.

\section{Author Contributions}

All authors contributed to data analysis, drafting or revising the article, gave final approval of the version to be published, and agree to be accountable for all aspects of the work.

\section{Disclosure}

The authors report no conflicts of interest in this work.

\section{References}

1. Ng SC, Shi HY, Hamidi N, et al. Worldwide incidence and prevalence of inflammatory bowel disease in the 21st century: a systematic review of population-based studies. Lancet. 2018;390(10114):27692778. doi:10.1016/S0140-6736(17)32448-0

2. Zielinska A, Salaga M, Wlodarczyk M, Fichna J. Focus on current and future management possibilities in inflammatory bowel diseaserelated chronic pain. Int $J$ Colorectal Dis. 2019;34(2):217-227. doi:10.1007/s00384-018-3218-0

3. Wu KC, Liang J, Ran ZH, et al. Chinese consensus on diagnosis and treatment of inflammatory bowel disease(Beijing, 2018). Chin J Pract Internal Med. 2018;38(9):796-813.

4. Shang HX, Wang AQ, Bao CH, et al. Moxibustion combined with acupuncture increases tight junction protein expression in Crohn's disease patients. World J Gastroenterol. 2015;21(16):4986-4996. doi:10.3748/wjg.v21.i16.4986

5. Zhou EH, Liu HR, Wu HG, et al. Down-regulation of protein and mRNA expression of IL-8 and ICAM-1 in colon tissue of ulcerative colitis patients by partition-herb moxibustion. Dig Dis Sci. 2009;54 (10):2198-2206. doi:10.1007/s10620-008-0620-4

6. Stein DJ. Massage acupuncture, moxibustion, and other forms of complementary and alternative medicine in inflammatory bowel disease. Gastroenterol Clin North Am. 2017;46(4):875-880. doi:10.1016/j.gtc.2017.08.015

7. Cheifetz AS, Gianotti R, Luber R, Gibson PR. Complementary and alternative medicines used by patients with inflammatory bowel diseases. Gastroenterology. 2017;152(2):415-429 e415. doi:10.1053/j. gastro.2016.10.004

8. Langhorst J, Wulfert H, Lauche R, et al. Systematic review of complementary and alternative medicine treatments in inflammatory bowel diseases. J Crohn's Colitis. 2015;9(1):86-106. doi:10.1093/ ecco-jcc/jju007

9. Ji J, Lu Y, Liu H, et al. Acupuncture and moxibustion for inflammatory bowel diseases: a systematic review and meta-analysis of randomized controlled trials. Evidence-Based Complementary Altern Med. 2013;2013:158352. doi:10.1155/2013/158352

10. Bao C, Wang D, Liu P, et al. Effect of electro-acupuncture and moxibustion on brain connectivity in patients with Crohn's disease: a resting-state fMRI study. Front Hum Neurosci. 2017;11:559. doi:10.3389/fnhum.2017.00559

11. Joos S, Brinkhaus B, Maluche C, et al. Acupuncture and moxibustion in the treatment of active Crohn's disease: a randomized controlled study. Digestion. 2004;69(3):131-139. doi:10.1159/000078151

12. Srinath A, Young E, Szigethy E. Pain management in patients with inflammatory bowel disease: translational approaches from bench to bedside. Inflamm Bowel Dis. 2014;20(12):2433-2449. doi:10.1097/ MIB.0000000000000170

13. Srinath AI, Walter C, Newara MC, Szigethy EM. Pain management in patients with inflammatory bowel disease: insights for the clinician. Therap Adv Gastroenterol. 2012;5(5):339-357. doi:10.1177/ $1756283 X 12446158$

14. Liu S, Hagiwara SI, Bhargava A. Early-life adversity, epigenetics, and visceral hypersensitivity. Neurogastroenterol Motil. 2017;29(9). doi:10.1111/nmo.12973

15. Anand P, Aziz Q, Willert R, van Oudenhove L. Peripheral and central mechanisms of visceral sensitization in man. Neurogastroenterol Motil. 2007;19(1 Suppl):29-46. doi:10.1111/j.1365-2982.2006.00873.x

16. Jain P, Hassan AM, Koyani CN, et al. Behavioral and molecular processing of visceral pain in the brain of mice: impact of colitis and psychological stress. Front Behav Neurosci. 2015;9:177. doi:10.3389/fnbeh.2015.00177 
17. Monteleone I, Marafini I, Dinallo V, et al. Sodium chloride-enriched diet enhanced inflammatory cytokine production and exacerbated experimental Colitis in mice. J Crohn's Colitis. 2017;11(2):237245. doi:10.1093/ecco-jcc/jjw139

18. Kawasaki Y, Kohno T, Zhuang ZY, et al. Ionotropic and metabotropic receptors, protein kinase $\mathrm{A}$, protein kinase $\mathrm{C}$, and Src contribute to C-fiber-induced ERK activation and cAMP response element-binding protein phosphorylation in dorsal horn neurons, leading to central sensitization. $J$ Neurosci. 2004;24(38):8310-8321. doi:10.1523/ JNEUROSCI.2396-04.2004

19. Huang Y, Yang YT, Liu XX, et al. Effect of herbal-partitioned moxibustion at Tianshu (ST 25) and Qihai (CV 6) on pain-related behavior and emotion in rats with chronic inflammatory visceral pain. J Acupuncture Tuina Sci. 2015;13(1):1-8. doi:10.1007/s11726-0150814-1

20. Zhou Q, Price DD, Caudle RM, Verne GN. Visceral and somatic hypersensitivity in TNBS-induced colitis in rats. Dig Dis Sci. 2008;53(2):429-435. doi:10.1007/s10620-007-9881-6

21. Morris GP, Beck PL, Herridge MS, Depew WT, Szewczuk MR, Wallace JL. Hapten-induced model of chronic inflammation and ulceration in the rat colon. Gastroenterology. 1989;96(3):795-803.

22. Al-Chaer ED, Kawasaki M, Pasricha PJ. A new model of chronic visceral hypersensitivity in adult rats induced by colon irritation during postnatal development. Gastroenterology. 2000;119(5):12761285. doi:10.1053/gast.2000.19576

23. Chaplan SR, Bach FW, Pogrel JW, Chung JM, Yaksh TL. Quantitative assessment of tactile allodynia in the rat paw. $J$ Neurosci Methods. 1994;53(1):55-63. doi:10.1016/0165-0270(94) 90144-9

24. Hargreaves K, Dubner R, Brown F, Flores C, Joris J. A new and sensitive method for measuring thermal nociception in cutaneous hyperalgesia. Pain. 1988;32(1):77-88. doi:10.1016/0304-3959(88) 90026-7

25. Araki Y, Andoh A, Fujiyama Y, Bamba T. Development of dextran sulphate sodium-induced experimental colitis is suppressed in genetically mast cell-deficient Ws/Ws rats. Clin Exp Immunol. 2000;119 (2):264-269. doi:10.1046/j.1365-2249.2000.01094.x

26. Kang S, Elf S, Lythgoe K, et al. p90 ribosomal S6 kinase 2 promotes invasion and metastasis of human head and neck squamous cell carcinoma cells. J Clin Invest. 2010;120(4):1165-1177. doi:10.1172/ JCI40582

27. Bhola NE, Thomas SM, Freilino M, et al. Targeting GPCR-mediated p70S6K activity may improve head and neck cancer response to cetuximab. Clin Cancer Res. 2011;17(15):4996-5004. doi:10.1158/ 1078-0432.CCR-10-3406

28. Zhang YM, Dai BL, Zheng L, et al. A novel angiogenesis inhibitor impairs lovo cell survival via targeting against human VEGFR and its signaling pathway of phosphorylation. Cell Death Dis. 2012;3:e406. doi:10.1038/cddis. 2012.145

29. Ford AC, Quigley EM, Lacy BE, et al. Effect of antidepressants and psychological therapies, including hypnotherapy, in irritable bowel syndrome: systematic review and meta-analysis. Am J Gastroenterol. 2014;109(9):1350-1365; quiz 1366. doi:10.1038/ajg.2014.148

30. Cooper TE, Fisher E, Gray AL, et al. Opioids for chronic non-cancer pain in children and adolescents. Cochrane Database Syst Rev. 2017;7:CD012538. doi:10.1002/14651858.CD003881.pub4

31. Tracey KJ. The inflammatory reflex. Nature. 2002;420(6917):853859. doi:10.1038/nature 01321

32. Fang JQ, Shao XM. New trains of thoughts about acupuncture analgesia-acupuncture analgesia feb involve multi-dimensional regulation of pain. Zhen Ci Yan Jiu. 2017;42(1):85-89.

33. Lu YJ, Cai XW, Zhang GF, et al. Long-term acupuncture treatment has a multi-targeting regulation on multiple brain regions in rats with Alzheimer's disease: a positron emission tomography study. Neural Regener Res. 2017;12(7):1159-1165. doi:10.4103/1673-5374.211197
34. Han JS. Acupuncture analgesia: areas of consensus and controversy. Pain. 2011;152(3 Suppl):S41-S48. doi:10.1016/j.pain.2010.10.012

35. Li X, Wang R, Shi X, et al. Reporting characteristics and quality of systematic reviews of acupuncture analgesia. Pain Pract. 2017;17 (8):1066-1074. doi:10.1111/papr.12555

36. Zhao JM, Lu JH, Yin XJ, et al. Comparison of electroacupuncture and moxibustion on brain-gut function in patients with diarrheapredominant irritable bowel syndrome: a randomized controlled trial. Chin J Integr Med. 2015;21(11):855-865. doi:10.1007/s11655015-2049-x

37. Bao C, Liu P, Liu H, et al. Different brain responses to electroacupuncture and moxibustion treatment in patients with Crohn's disease. Sci Rep. 2016;6:36636. doi:10.1038/srep36636

38. Li ZY, Huang Y, Yang YT, et al. Moxibustion eases chronic inflammatory visceral pain through regulating MEK, ERK and CREB in rats. World J Gastroenterol. 2017;23(34):6220-6230. doi:10.3748/ wjg.v23.i34.6220

39. Li ZY, Yang YT, Hong J, et al. Extracellular signal-regulated kinase, substance $\mathrm{P}$ and neurokinin-1 are involved in the analgesic mechanism of herb-partitioned moxibustion. Neural Regener Res. 2017;12 (9):1472-1478. doi:10.4103/1673-5374.215259

40. Gao YJ, Zhang L, Samad OA, et al. JNK-induced MCP-1 production in spinal cord astrocytes contributes to central sensitization and neuropathic pain. J Neurosci. 2009;29(13):4096-4108. doi:10.1523/ JNEUROSCI.3623-08.2009

41. Ji RR, Befort K, Brenner GJ, Woolf CJ. ERK MAP kinase activation in superficial spinal cord neurons induces prodynorphin and NK-1 upregulation and contributes to persistent inflammatory pain hypersensitivity. $J$ Neurosci. 2002;22(2):478-485.

42. Ji RR, Woolf CJ. Neuronal plasticity and signal transduction in nociceptive neurons: implications for the initiation and maintenance of pathological pain. Neurobiol Dis. 2001;8(1):1-10. doi:10.1006/ nbdi. 2000.0360

43. Gao YJ, Xu ZZ, Liu YC, Wen YR, Decosterd I, Ji RR. The c-Jun $\mathrm{N}$-terminal kinase 1 (JNK1) in spinal astrocytes is required for the maintenance of bilateral mechanical allodynia under a persistent inflammatory pain condition. Pain. 2010;148(2):309-319. doi:10.1016/j.pain.2009.11.017

44. Zhao YJ, Li JH, Hu B, et al. Extracellular signal-regulated kinase activation in the spinal cord contributes to visceral hypersensitivity induced by craniofacial injury followed by stress. Neurogastroenterol Motil. 2018;30(2). doi:10.1111/nmo.13161.

45. Kondo T, Sakurai J, Miwa H, Noguchi K. Activation of p38 MAPK through transient receptor potential A1 in a rat model of gastric distension-induced visceral pain. Neuroreport. 2013;24(2):68-72. doi:10.1097/WNR.0b013e32835c7df2

46. Broom OJ, Widjaya B, Troelsen J, Olsen J, Nielsen OH. Mitogen activated protein kinases: a role in inflammatory bowel disease? Clin Exp Immunol. 2009;158(3):272-280. doi:10.1111/j.1365-2249.20 09.04033.x

47. Ju H, Feng Y, Zhou J, Cui ZR, Gao ZF, Wang S. MAPK pathway protein expression in acute colitis visceral pain model analgesic effect of morphine. Chin J Pain Med. 2014;20(3):139-144.

48. Zhao JM, Li L, Chen L, et al. Comparison of the analgesic effects between electro-acupuncture and moxibustion with visceral hypersensitivity rats in irritable bowel syndrome. World $J$ Gastroenterol. 2017;23(16):2928-2939. doi:10.3748/wjg.v23.i16.2928

49. Zhao JM, Lu JH, Yin XJ, et al. Comparison of electroacupuncture and mild-warm moxibustion on brain-gut function in patients with constipation-predominant irritable bowel syndrome: a randomized controlled trial. Chin J Integr Med. 2018;24(5):328-335. doi:10.1 007/s11655-018-2838-0

50. Zhu Y, Wu Z, Ma X, et al. Brain regions involved in moxibustioninduced analgesia in irritable bowel syndrome with diarrhea: a functional magnetic resonance imaging study. BMC Complement Altern Med. 2014;14:500. doi:10.1186/1472-6882-14-500 
51. Wu HG, Yan J, Yu SG, et al. Research current situation and development trend of of moxibustion therapy. Shanghai J Acu-Mox. 2009;28(01):1-6.

52. Wu HG, Weng ZJ, Liu HR, et al. Analgesic and anti- inflammatory effects of moxibustion on immune-related disease. World Chin Med. 2016;11(12):2505-2514,2520.

53. Li J, Jiang J. Role of transient receptor potential vanilloid subtype 1 in the increase of thermal pain threshold by moxibustion. $J$ Tradit Chin Med. 2015;35(5):583-587.
54. Liao F, Zhang C, Bian Z, et al. Characterizing heat-sensitization responses in suspended moxibustion with high-density EEG. Pain Med. 2014;15(8):1272-1281. doi:10.1111/pme.12512

55. Zheng B, Hu L, Song X, et al. Analgesic effect of different moxibustion durations in rheumatoid arthritis rats. J Tradit Chin Med. 2014;34(1):90-95.

56. Chen S, Wang S, Rong P, et al. Acupuncture for visceral pain: neural substrates and potential mechanisms. Evid Based Complement Alternat Med. 2014;2014:609594. doi:10.1155/2014/105186

\section{Publish your work in this journal}

The Journal of Pain Research is an international, peer reviewed, open access, online journal that welcomes laboratory and clinical findings in the fields of pain research and the prevention and management of pain. Original research, reviews, symposium reports, hypothesis formation and commentaries are all considered for publication. The manuscript management system is completely online and includes a very quick and fair peer-review system, which is all easy to use. Visit http:// www.dovepress.com/testimonials.php to read real quotes from published authors. 